\title{
Analysis on Feasibility and Study of Machinery Protection Cover
}

\author{
Zhao Zhanfeng \\ Wenzhou Vocational \& Technical College, Wenzhou. 325035 \\ Zhanfeng @qq.com
}

Keywords: Machinery, Facilities, Design, Analysis.

\begin{abstract}
A machinery protection cover, includes the first splicing cover, the second splicing cover, the third splicing cover, the fourth splicing cover, orbit and central control circuit. The smart electronic control splicing among the first splicing cover, the second splicing cover, the third splicing cover and the fourth splicing cover, has the advantages of simple operation that can either machinery work in safe or offer safe working situation to manufacturing work with less disassemble and instalment time so as to improve work efficiency.
\end{abstract}

\section{Technological Analysis}

During the work of large-scale machinery, the surroundings of the machinery need protective cover to protect it. On one hand, to make sure the normal work of the machinery; on the other hand, to make sure the working staff in safety and to prevent touch between working staff and the working machinery. In particular, it is necessary to set protective cover in the situation of highly work and low cost of large-scale machinery.

At present, the existing large-scale machinery protective cover is replaced and installed with steel structure and steel wire gauze. When workers need to maintain and change parts, maintain and conserve facilities, and regularly check, quantities of workers and work time would be in need to disassemble and install the protective cover, which will cause low work efficiency, bad flexibility and poor smart function.

Hence, a machinery protective cover should be in need of design to complete smart electronic control splicing and instalment with simple operation steps.

\section{Analysis on Feasibility}

The first splicing cover, the second splicing cover, the third splicing cover, the fourth splicing cover, orbit and central control circuit, and the first splicing cover, the second splicing cover, the third splicing cover, the fourth splicing cover separately connected to the smoothing orbit; The first splicing cover, the second splicing cover, the third splicing cover, the fourth splicing cover separately include the first side lever, second side lever, top lever, upright lever, bottom lever, first mounting lever, second mounting lever, mounting plate, traveling motor, brake, one side of the rod, the second side of the rod and the end of the top rod connected to form a triangular top lever structure. The side is installed limit switch and electromagnet attached both the first side lever of the first splicing cover and the second side lever of the second splicing cover. The side is installed limit switch and electromagnet attached both the first side lever of the second splicing cover and the second side lever of the third splicing cover. The side is installed limit switch and electromagnet attached both the first side lever of the third splicing cover and the second side lever of the fourth splicing cover. The side is installed limit switch and electromagnet attached both the first side lever of the fourth splicing cover and the second side lever of the first splicing cover. Both ends of the top lever separately are connected with two upright levers, and the other ends of the two upright levers separately are connected with the two ends of the bottom lever. The top lever, two upright levers and the bottom lever constitute a rectangle enclosure structure. At least three groups of the first mounting lever are connected between the bottom lever and the second mounting lever, among of 
which the first mounting lever is fixed contact rollers that can roll on orbits. Brakes are fixed on one of the contact rollers. Mounting plates are fixed between the first mounting level and the stated bottom lever. Running motor is fixed on the mounting plate and the running motor is connected with one group of contact rollers by Axis. The triangle top bar structure and rectangle enclosure structure are separately fixed nets.

\section{Assembly Design}

The first side lever is the same in length as the second side lever. The triangle top bar structure is isosceles right triangle structure, which can make the first splicing cover, the second splicing cover, the third splicing cover, the fourth splicing cover is arranged and fixed from the beginning to the end to form the rectangle protective cover, as Fig.1 shows.

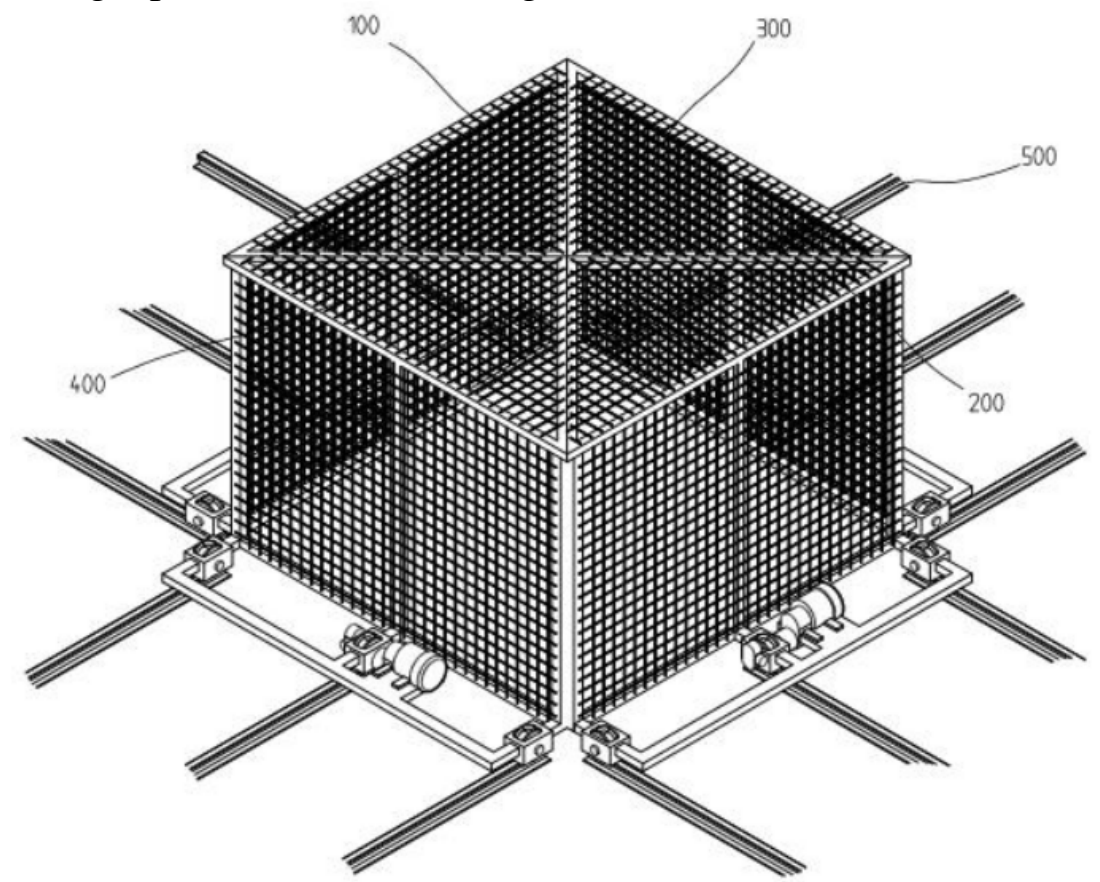

Fig.1 Rectangle Protective Cover

The orbit is set on the steel plate or the wooden plate, and then the steel plate or the wooden plate is fixed onto the groundwork. The upright lever and the first mounting lever is formed to all-in-one structure as "L" structure. The first side lever and the second side lever take on 90 degree settings with the upright lever. The first mounting lever and the first side lever, the second lever is set in opposite directions, as Fig.2 shows. 


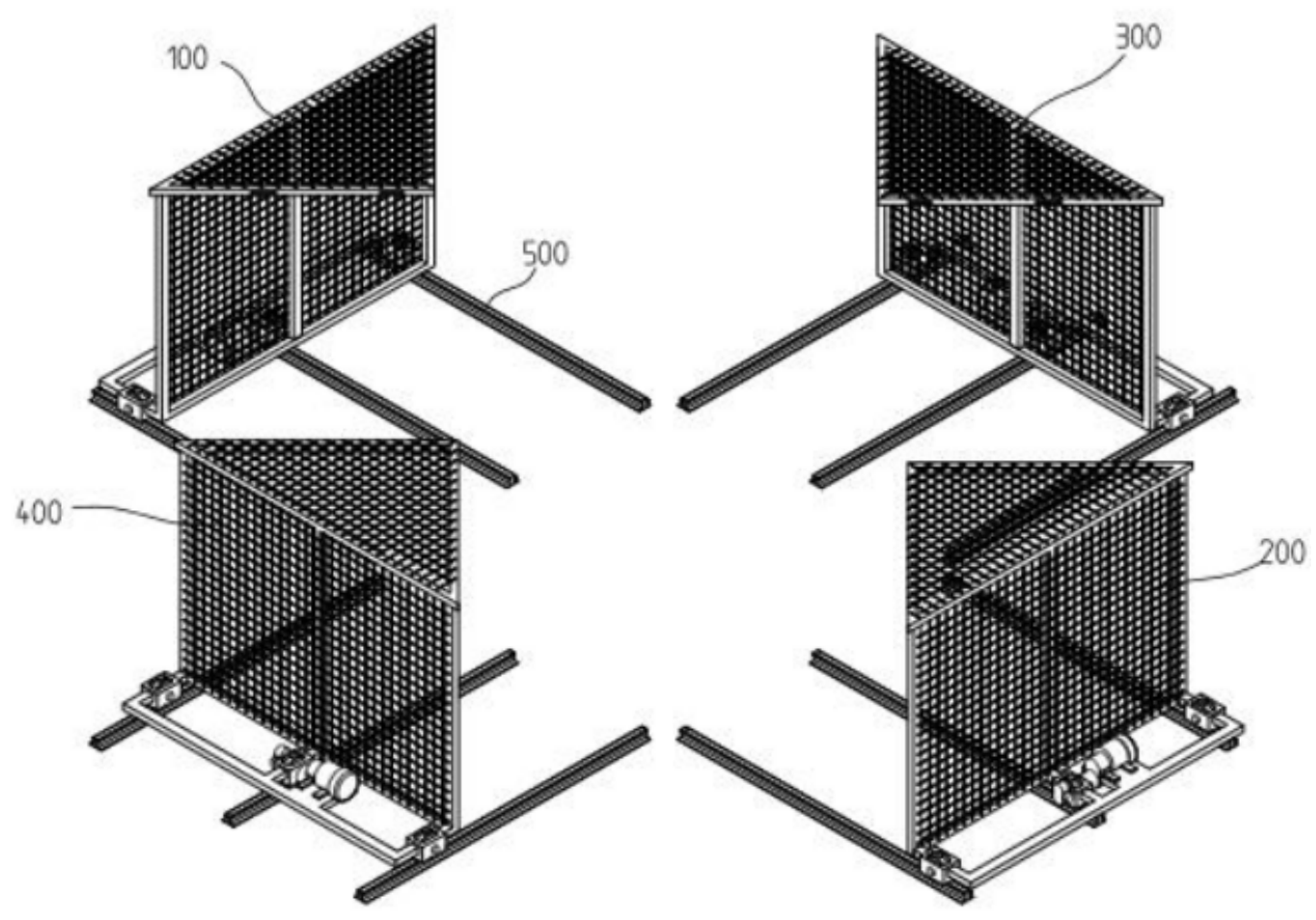

Fig. 2 "L" Structure

The central control circuit includes limit switch's checking circuit referring to limit switches, judging whether electromagnet is working electromagnet drive circuit, judging whether running motor is the working running motor drive circuit, judging whether the brakes are working brakes drive circuit and central controller. As Fig.3 shows.

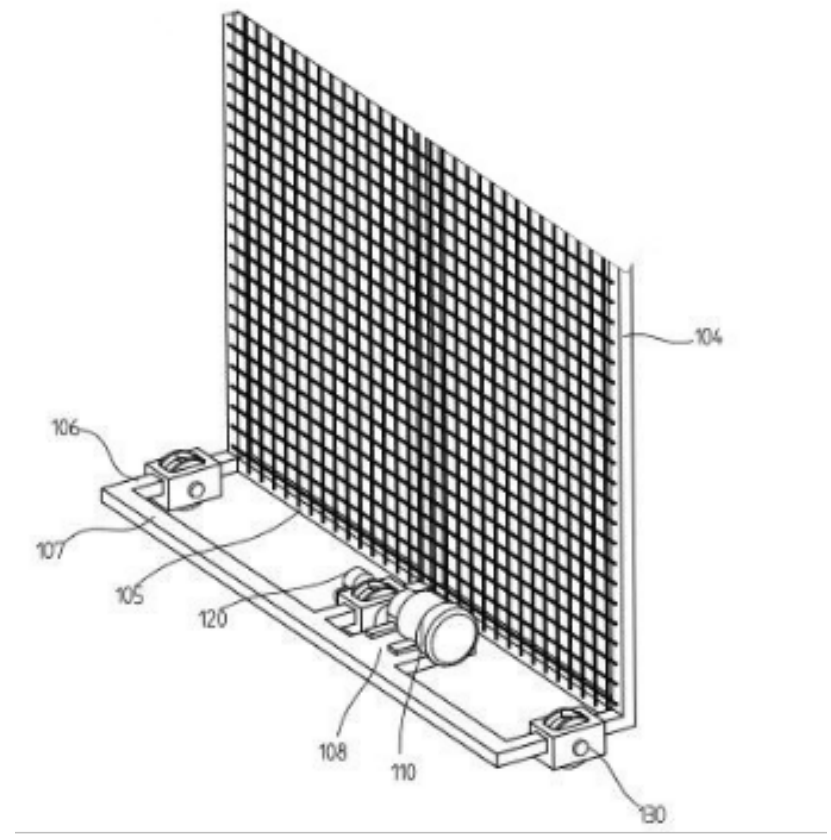

Fig.3 Circuit and Central Controller

The beneficial effect is in that it is convenient to operate by the smart electronic control splicing among the first splicing cover, the second splicing cover, the third splicing cover and the fourth splicing cover. It can either guarantee the machinery work in safety or offer workers with a safe working surroundings with less disassemble and instalment time so as to improve work efficiency. As Fig. 4 shows. 


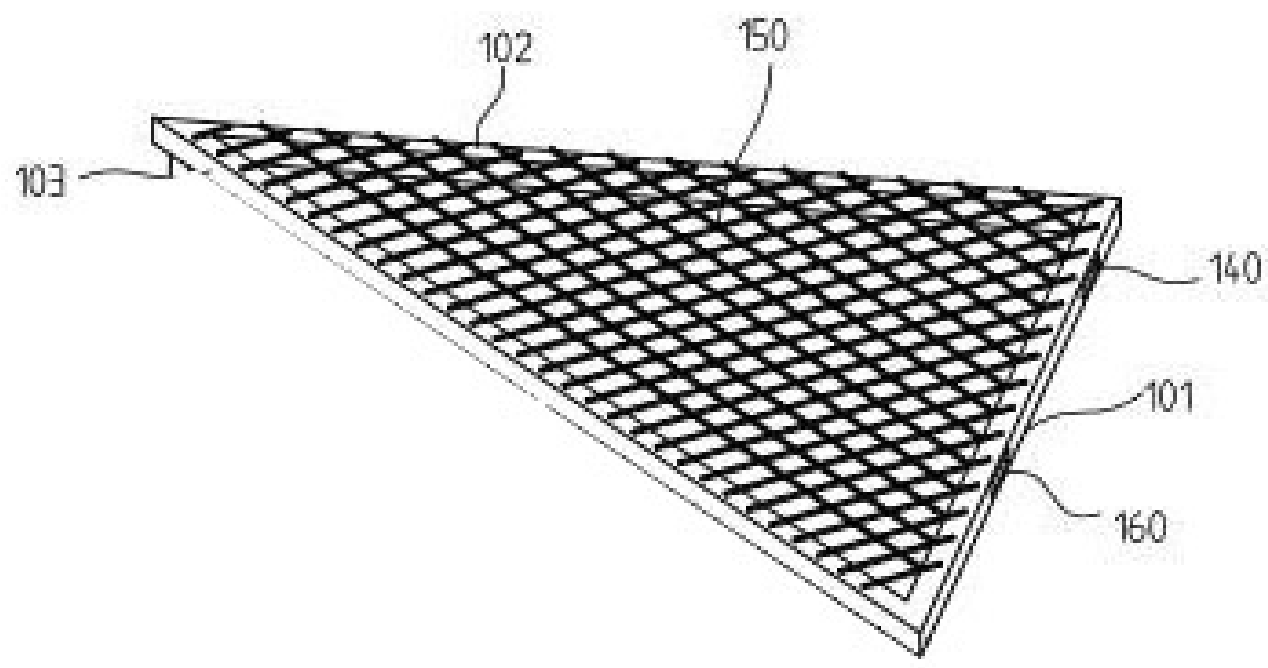

Fig. 4 Splicing Cover

The first side lever 101 is the same in length as the second side lever. The triangle top bar structure is an isosceles right triangle structure that can make the first splicing cover 100, the second splicing cover 200, the third splicing cover 300 and the fourth splicing cover 400 to form the rectangle protective cover from the beginning to the end. Thus, the objective of the machinery can be protected and the workers can avoid touch in the situation of the machinery working.

\section{Conclusion}

The second side lever 102 of the first splicing cover 100 is touched in accordance to the theory above. Hence, the objective of achieving install smart protective covers can be reached with the work methods above when separated protective covers are in need.

\section{References}

[1] ZHAO Hua, CAO Guo-zhong. Study on Conceptual Design Process Based on the Biological Effects[J].Machine Design Research, 2011, 27(3): 105-110.

[2]TAN Ao-nan, LI Hong-hai. Emotional Design Research Literature Review[J]. Design, 2016, 29(1): 74-75.

[3]AN Xiao-ya, YANG Yun, LIU Ping-zhi. Similarity Measuring of Spatial pological Relations Based on Topological Predication[J]. Journal of Geo-information ience, 2013(2): 159—165.

[4]LIU Wei, CAO Guo-zhong, TAN Run-hua. Research on Method for Coding Multi ical Effects[J]. Chinese Journal of Engineering Design, 2014, 21(1): 1—5.

[5]ZHOU Yang, ZHANG Yu-hong. Analysis of Memory in Emotional Design[J]. ackaging Engineering, 2014, 35(4): 70—74.

[6]LIU Wei, CAO Guo-zhong, TAN Run-hua. Research on Measures to Technical zation of Multi Biolog $\square$ ical Effects[J]. Journal of Mechanical Engineering,2016(9): 129-140. 